\title{
Association of vesicoureteral reflux and renal scarring in urinary tract infections
}

\author{
İsa Yılmaz, M.D. ${ }^{a}$, Harun Peru, M.D., Professor ${ }^{b}$, Fatma H. Yllmaz, M.D ${ }^{a}$, \\ Tamer Sekmenli, M.D. Assistant professorc ${ }^{c}$ İlhan Çiftçi, Associate professorc and \\ Fatih Kara, Assistant professor ${ }^{d}$
}

\begin{abstract}
Introduction. The aim was to investigate the relationship between vesicoureteral reflux (VUR) and renal damage in non-febrile, febrile for the first time and recurrent urinary tract infection (UTI) patients. The secondary aim was to determine whether C-reactive protein (CRP) in febrileUTIs could be a predictor of renal scarring. Population and methods. This prospective study included non-febrile, febrile for the first time and recurrent pediatric UTI cases. The routine lab analyses comprised a complete blood count, urea, creatinine, fully automated urinalysis, urine culture and CRP analyses. All the participants were examined using urine ultrasonography subsequent to their UTI diagnosis, voiding cystourethrography (VCUG) after six weeks and Tc-99m dimercaptosuccinic acid (DMSA) static renal scintigraphy after six months.

Results. There were included 47 children with non-febrile UTIs, 48 with first febrile UTIs and 61 with recurrent UTIs. A statistically significant difference was found among the groups in terms of VUR and renal scarring $(\mathrm{p}=0.001$ and $p=0.011$, respectively). A statistically significant difference was also found in terms of renal scarring between patients with and without VUR $(p=0.001)$. Moreover, a statistically significant difference was also present in relation to renal scarring $(p<0.05)$ in patients with five-fold lower or higher CRP values than the accepted cut-off value $(5 \mathrm{mg} / \mathrm{dl})$.

Conclusion. The ratio of renal scars detected was found to be parallel to the VUR frequency. The higher the VUR grade, the more renal damage was found. A positive correlation between elevated CRP and renal scarring was determined, indicating the presence of scarring during the diagnosis of pyelonephritis.

Key words: vesico-ureteral reflux, child, renal scarring, urinary tract infections.
\end{abstract}

http:/ / dx.doi.org/10.5546/ aap.2018.eng.e542

E-mail address:

İsa Y1lmaz M.D.:

drisayilmaz@hotmail. com

Funding:

None

Conflict of interest:

None.

Received: 10-19-2017

Accepted: 2-19-2018
To cite: Yilmaz I, Peru H, Yilmaz FH, et al Association of vesicoureteral reflux and renal scarring in urinary tract infections. Arch Argent Pediatr 2018;116(4):e542-e547.

\section{INTRODUCTION}

Urinary tract infections (UTIs) are, especially in children under the age of three, among the most common bacterial infections. ${ }^{1}$ In $10-64 \%$ of acute pyelonephritis (APN) cases, renal parenchymal damage is reported, with recurrent and improperly treated cases exhibiting morbidity risks such as hypertension and chronic renal failure (CRF). ${ }^{2}$

Vesicoureteral reflux (VUR) is the most common anomaly detected in paediatric UTI cases, with an incidence of $30-50 \%$, while the presence of renal scarring is detected in $30-40 \%$ of cases. ${ }^{3,4}$ Therefore, every paediatric UTI case should be investigated for VUR. Dimercaptosuccinic acid (DMSA) scintigraphy is currently the best imaging technique available for acute pyelonephritis-associated renal injuries. ${ }^{5}$ Renal injury, as detected by DMSA scintigraphy during or after acute pyelonephritis, is reported to be closely associated with clinical and laboratory findings at the onset of the disease. $^{6}$

Despite the availability of advanced examination and treatment techniques, the close follow-up of early age infections is important in cases of UTI, since serious complications may occur. The aim of the present study was to investigate the relationship between VUR and renal injury in initially non-febrile, febrile for the first time and recurrent UTI patients. The secondary aim was to determine whether $\mathrm{C}$-reactive protein (CRP) in febrile UTIs could be a predictor of renal scarring. 


\section{POPULATION AND METHODS}

This study was designed prospectively in the Paediatric Nephrology section of the Department of Pediatrics at Selçuk University. The original study included non-febrile, febrile for the first time and recurrent UTI patients. The present study was approved by the Ethics Committee of Selçuk University. Written informed consent was obtained from each participant prior to enrolment.

UTI patients aged between $0-16$ years who had been diagnosed with laboratory findings such as a complete blood count, ESR, CRP, urine culture studies, urinary system ultrasonography, voiding cystourethrography (VCUG) and Tc-99m DMSA scintigraphy were included in the study. The exclusion criteria were congenital anomalies, neurological diseases, obstructive urological anomalies and systemic diseases.

The UTI patients were classified as either nonfebrile (Group 1), first febrile episode (Group 2) or recurrent UTIs (Group 3).

The participants' complete blood count, urea, creatinine, fully automated urinalysis, urine culture, ESR and CRP were evaluated. A clean catch urine sample was obtained from patients who were toilet trained, while a urine catheter was used for patients who were not toilet trained.

Cystitis was considered in patients whose body temperature, white blood cell, sedimentation and CRP values were within normal ranges. All the patients had one or more of the symptoms of dysuria, stranguria, frequency, urgency, malodorous urine, incontinence, haematuria and suprapubic pain, and there was growth in the urine culture. Until clean urine cultures were achieved, oral antibiotics constituted the only treatment modality. Pyelonephritis was considered in patients with a body temperature of $\geq 38.5^{\circ} \mathrm{C}$, sedimentation of $\geq 35 \mathrm{~mm} /$ hour, a CRP value of $\geq 5$ times the cut-off value and growth in their urine culture. Intravenous antibiotics were administered for at least 48 hours after the feverless period until the urine cultures were clear and the CRP values were negative, with subsequent oral antibiotics being prescribed for 14 days. The patients who were febrile for the first time and those with recurrent UTIs took a prophylactic oral antibiotic prior to VCUG. Prophylaxis continued for at least six months for those diagnosed with VUR.

In addition, along with the UTI diagnosis, urinary ultrasonography was performed for all participants, while VCUG was performed six weeks later. In terms of the VCUG, bladder catheterisation was performed using a Foley catheter according to the age of the patients. Radiographic reflux was assessed using the international reflux rating system. Grade 1 is considered if the contrast agent is only seen in the ureter, Grade 2 if it is up to the renal calyces but the ureter is not dilated, Grade 3 if the ureter and renal pelvis are mildly dilated but the calyces are yet not blunted, Grade 4 if the ureters are mild tortuous, the renal pelvis dilated and the calyces are blunted, and Grade 5 if the ureters are significantly tortuous and the renal pelvis and calyces are highly dilated.

After six months, the patients' static renal scintigraphies were obtained via DMSA. The patients had been followed up monthly in the polyclinic by conducting full-automatic urinalysis and urine culture. The urine cultures of all the patients were sterile until the VCUG and Tc- $99 \mathrm{~m}$ DMSA static renal shots.

\section{Statistical analyses}

SPSS-15 software (SPSS Inc., Chicago, Illinois, USA) was used for all statistical analyses. The descriptive data were presented as the mean \pm SD. Kolmogorov-Smirnov and ShapiroWilk normality tests were performed in order to determine whether the data were appropriate for a normal distribution. All parametric data were analysed using Student's t-test, while nonparametric data were analysed with the chisquare test and the Mann-Whitney $U$ test with the Bonferroni correction. The Kruskal-Wallis test was used to compare the groups. The threshold level for statistical significance was $\mathrm{p}<0.05$.

\section{RESULTS}

There were included 47 children diagnosed with non-febrile UTIs (Group 1), 48 with first febrile UTIs (Group 2) and 61 with recurrent UTIs (Group 3) (Figure 1).

The mean age and distribution of the participants according to gender are presented in Table 1. While no statistically significant difference was determined between Group 1 and Group 2 in terms of age ( $p>0.05)$, there was a statistically significant difference between Group 3 and the other two groups $(p=0.001)$. Bacterial growth in the urine culture of all the groups included $73 \%$ E. coli, 10\% Klebsiella, 8\% Enterococcus, 5\% Proteus, 3\% Pseudomonas and 1\% Enterobacter. The mean laboratory results of the patients are given in Table 2. Compared to Group 
1, the white blood cell, CRP and sedimentation values of Group 2 and Group 3 were higher and statistically significant $(p=0.001)$.

A statistically significant difference was detected between the patients with and without VUR in terms of renal scarring $(p=0.001)$. Renal scarring was detected in $38 \%$ of patients with mild reflux and $73 \%$ of those with severe reflux (Table 3).

During the VCUG, VUR was determined in $17 \%$ of patients in Group 1, 39\% in Group 2 and $62 \%$ in Group 3. DMSA scintigraphy showed renal scarring in $13 \%$ of patients in Group 1, $31 \%$ in Group 2 and 39\% in Group 3. Among

FIGURE 1. Flow chart of patient inclusion

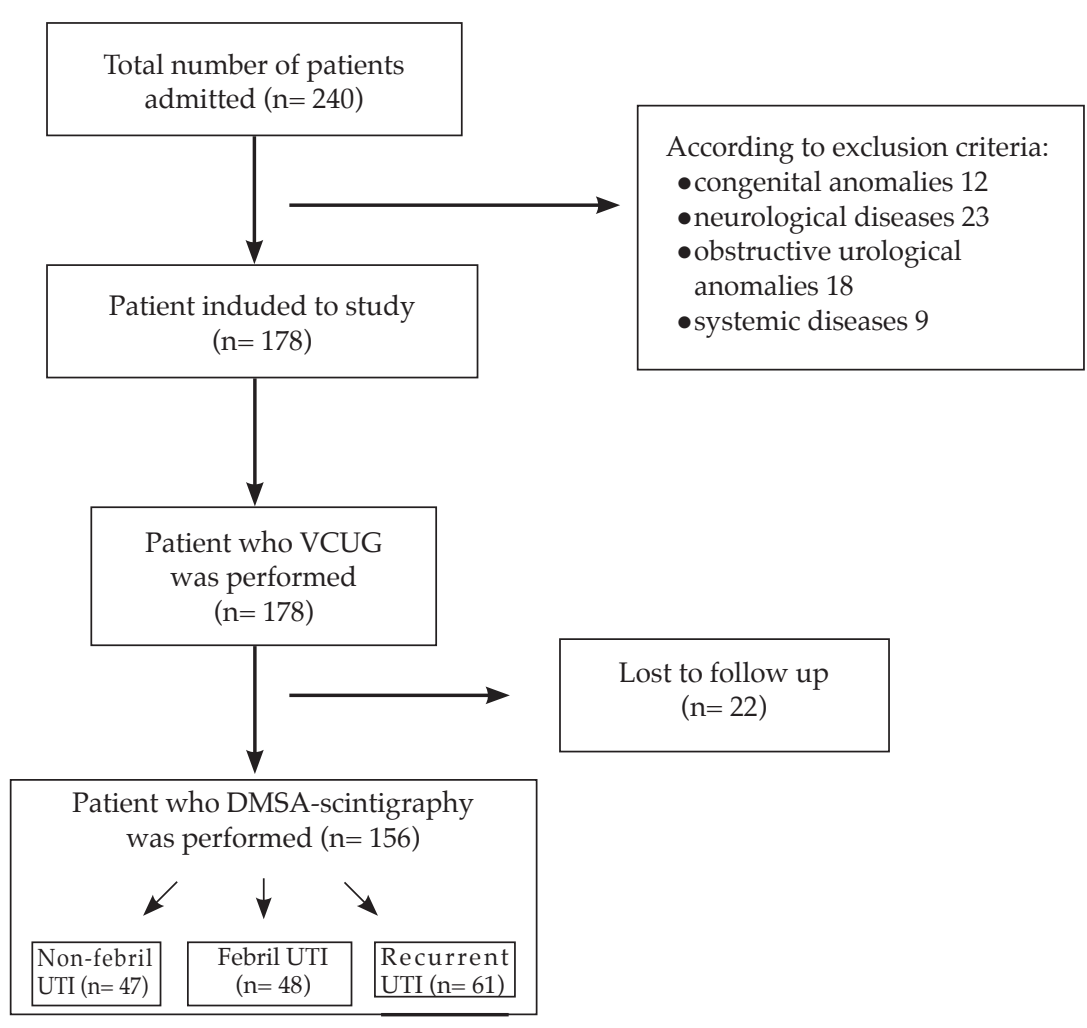

TABLE 1. Demographic characteristics of groups with VUR and renal scar distributions

\begin{tabular}{lcccc}
\hline & $\begin{array}{c}\text { Group 1 } \\
(\mathbf{n}=\mathbf{4 7})\end{array}$ & $\begin{array}{c}\text { Group 2 } \\
(\mathbf{n}=\mathbf{4 8})\end{array}$ & $\begin{array}{c}\text { Group 3 } \\
(\mathbf{n = 6 1 )}\end{array}$ & $p$ \\
\hline Age (months) (mean \pm SD) & $41,5 \pm 48,3$ & $48,5 \pm 35,82$ & $81,72 \pm 39,9$ & 0,001 \\
Female/male (n) & $32 / 15$ & $40 / 8$ & $53 / 8$ & - \\
VUR (\%) & 17 & 39 & 62 & 0,001 \\
Renal scar $(\%)$ & 13 & 31 & 39 & 0,011 \\
\hline
\end{tabular}

TABLE 2. Correlation of the groups' laboratory results

\begin{tabular}{lcccc}
\hline & Group 1 & Group 2 & Group 3 & $p$ \\
\hline White bloodcell $\left(/ \mathrm{mm}^{3}\right)$ & $9724,8 \pm 3537,67$ & $17992,56 \pm 7578,97$ & $12407,14 \pm 5526,65$ & 0,001 \\
Sedimentation $(\mathrm{mm} / \mathrm{h})$ & $8,65 \pm 11,65$ & $43,94 \pm 20,29$ & $30,33 \pm 24,15$ & 0,001 \\
CRP $(\mathrm{mg} / \mathrm{L})$ & $5,21 \pm 4,48$ & $80,92 \pm 55,18$ & $46,05 \pm 55,43$ & 0,001 \\
\hline
\end{tabular}


the groups, a statistically significant difference emerged in terms of the VUR and presence of renal scarring. The $\mathrm{p}$ values were 0.001 and 0.011 , respectively (Table 3). Some $17 \%$ of patients in all the groups did not present with VUR; however, $56 \%$ of the VUR patients had renal scarring as shown via DMSA scintigraphy. There was a statistically significant difference in renal scarring between patients with and without VUR $(p=0.001)$. Renal scaring was detected in $38 \%$ of patients with mild reflux and $73 \%$ of patients with severe reflux (Table 3).

While renal scarring was detected in $14 \%$ of patients in Group 1 with negative CRP levels, it was detected in $32 \%$ of patients in Group 2 with positive CRP levels. A statistically significant difference was found between the CRP values of the positive and negative patients $(\mathrm{p}<0.0001)$ (Table 3).

\section{DISCUSSION}

Throughout the treatment of UTIs, which represent a common type of childhood infection, the diagnosis of VUR during the early stages is an important approach for preventing kidney damage. VUR nephropathy and recurrent attacks of pyelonephritis result in end-stage renal disease. ${ }^{2}$ When compared to males, females are more prone to UTIs due to anatomical differences. Among the three groups involved in the present study, which included 156 patients, the proportion of females was, in line with the relevant literature, higher. ${ }^{6}$ Therefore, we believe that urinary evaluation is necessary even if fever is diagnosed with a different focus in female patients.

Gram (-) bacteria, especially E. coli, are the most frequently isolated pathogenic agents described in the literature. Indeed, E. coli growth is reportedin $80-90 \%$ of acute UTIs. ${ }^{7}$ In line with the relevant literature, in the present study, E. coli was the most commonly isolated agent.

High levels of white blood cells, ESR and CRP are important predictors of cystitis pyelonephritis.

TABLE 3. Correlation of renal scar with VUR and CRP values

\begin{tabular}{lccc}
\hline & Renal scar (-) & Renal scar (+) & $p$ \\
\hline $\operatorname{VUR}(-)(\mathrm{n}=78)$ & 65 & 13 & 0,001 \\
$\operatorname{VUR}(+)(\mathrm{n}=52)$ & 23 & 29 & \\
$\operatorname{CRP}(-)(\mathrm{n}=29)$ & 25 & 4 & $<0,0001$ \\
$\operatorname{CRP}(+)(\mathrm{n}=47)$ & 32 & 15 & \\
\hline
\end{tabular}

Ayazi et al., compared the CRP, ESR and white blood cell counts with the urine culture results, that is, the "gold standard" for diagnosis, and concluded that if these three parameters were studied together and evaluated alongside the physical examination and patient history, they are suggestive of the localisation of UTI patients ${ }^{8}$. In the present study, the comparison of the white blood cell, ESR and CRP values in the patients in Group 1 showed that these counts were lower, which was as expected. The counts were higher in the first febrile group when compared with Group 3, that is, the recurrent UTIs group. The UTI level was higher in two patients than in the recurrent group. This result was attributed to the fact that some recurring UTIs in the patients in Group 3 were not always seen in the form of acute pyelonephritis as they could also appear as cystitis.

VUR, a common urinary system anomaly seen in children, can be present in a wide range of clinical stages, ranging from spontaneous healing to recurrent pyelonephritis episodes with kidney scarring and, finally, CRF. In many clinical studies, the relation between UTIs, VUR and renal scarring has been established. Thus, in one in three UTI patients, as well as in one in three VUR patients, renal scarring is present. ${ }^{9}$ The incidence of VUR in patients presenting with UTIs varies between races. Gelfand et al., determined the VUR frequency to be 32\%, while Mahyar et al., found it to be $39.2 \%{ }^{4,10}$ According to the prior literature, the highest VUR rates range between $41 \%$ and $63 \%$ in the USA, the UK and Italy, with the lowest rates being 6-12\% in African American children and $10 \%$ in Jamaican children., ${ }^{3,1-14}$ In our country, in a study involving 300 patients, Yilmaz et al. determined that the VUR rate was $30.9 \% .{ }^{15}$ In the present study, the VUR rate was found to be in accordance with the relevant literature as well as statistically significant when the groups were examined separately. The higher reflux rate, especially in the patients in Group 3, can be attributed to the fact that the study was conducted in a tertiary healthcare centre and hence the referred patients had better and more frequent follow-up opportunities.

Among the prior studies defining the relation between renal scarring and VUR, the study conducted by Caino et al., reported the presence of renal scarring to be $67 \%$ in VUR cases and $16 \%$ in cases without VUR. ${ }^{16}$ Lee et al., reported that refluxes in children increased the risk of renal scarring; however, they did not correlate the 
VUR grade and scarring. ${ }^{17}$ Peru et al., reported a high renal scarring rate $(37.1 \%)$ in grade 1 VUR patients and a higher renal scarring rate $(61.5 \%)$ in patients with grades $4-5$ VUR. In the same study, $22.7 \%$ VUR and $9.9 \%$ renal scarring were detected in patients admitted with first nonfebrile UTIs. ${ }^{18}$ The present study determined a statistically significant difference in terms of renal scarring in patients with and without VUR. In line with the relevant literature, the present study supports the finding that more renal scars are present in patients with VUR. Further, the presence of $16 \%$ reflux and $13 \%$ renal scarring in the first non-febrile UTI group highlights the importance of close follow-up and prophylactic treatment. Another outcome of the present study is, in accordance with the relevant literature, the finding that more renal scars are present in patients with severe reflux (grades 4-5).

The most commonly preferred method of imaging during and after febrile UTI diagnosis is USG. In this regard, the American Academy of Pediatrics' 2011 guidelines include a recommendation for the use of USG imaging in 2- to 24-month-old children after febrile UTIs. Bush et al. found renal scarring on DMSA scintigraphy in 99 out of 512 patients following febrile UTIs with normal USG, and they concluded that USG had only low renal damage detection power. ${ }^{19}$ Another meta-analysis determined, as compared to DMSA scintigraphy, that USG had a sensitivity rate of $37-100 \%$ and a specificity of $65-99 \%$ due to the fact that USG is dependent on personal interpretation. ${ }^{20}$ Similar to the findings of numerous prior studies, the imaging power of USG was determined to be weak in the present study.

The CRP value is important in the discrimination of UTI localisation as well as being among the accepted risk factors for the development of parenchymal damage. Studies have shown that the CRP is significantly higher at the time of admission when renal damage is detected using DMSA scintigraphy following pyelonephritis. ${ }^{21-24}$ Likewise, in the present study, more renal scars were detected in patients with positive CRP values, with a statistically significant difference being found between such patients and those with negative CRP values $(\mathrm{p}<0.0001)$.

\section{CONCLUSIONS}

The renal scar ratio is higher in patients with febrile and recurrent UTIs than in those with non-febrile UTIs. Nevertheless, even in patients experiencing their first non-febrile UTIs, renal scarring as determined with DMSA scintigraphy is found at a considerable rate. The renal scar ratio detected in these patients is parallel to the VUR frequency. In other words, the higher the VUR grade, the more renal scarring is found. CRP elevation in the diagnosis of pyelonephritis is positively correlated with renal scarring, while laboratory parameters are suggestive of the presence of renal scarring.

\section{Acknowledgements}

The authors wish to thank all the study participants for their contribution to the project.

\section{REFERENCES}

1. Shaw KN, Gorelick M, McGowan KL, et al. Prevalence of urinary tract infection in febrile young children in the emergency department. Pediatrics 1998;102(2):e16.

2. Bensman A, Durand O, Ulnski T. Urinary Track Infections. In: Avner E, Harmon W, Niaudet P, et al (Ed). Pediatric Nephrology. 6th ed. Berlin: Springer; 2009.P.1299-11.

3. Melhem RE, Harpen MD. Ethnic factors in the variability of primary vesico-ureteral reflux with age. Pediatr Radiol 1997;27(9):750-1.

4. Cooper CS, Austin JC. Vesicoureteral reflux: who benefits from surgery? Urol Clin North Am 2004;31(3):535-41.

5. Jaksic E, Bogdanovic R, Artiko V, et al. Diagnostic role of initial renal cortical scintigraphy in children with the first episode of acute pyelonephritis. Ann Nucl Med 2011; 25(1):37-43.

6. Spencer DJ, Schwaderer A, McHugh K, et al. Pediatric urinary tract infections: an analysis of hospitalizations, charges, and costs in the USA. Pediatr Nephrol 2010; 25(12):2469-75

8. Alon US. Urinary Tract Infection and Perinephric/ Intranephric Abscess. Burg FD. In: Burg F, Polin RA, Gershon AA, et al (Ed). Current Pediatric Therapy. 18th ed. Michigan: Saunders Elsevier; 2006.P.594-6.

9. Ayazi P, Mahyar A, Daneshi MM, et al. Diagnostic accuracy of the quantitative c-reactive protein, erythrocyte sedimentation rate and white blood cell count in urinary tract infections among infants and children. Malays J Med Sci 2013;20(5):40-6.

10. Gelfand MJ, Koch BL, Elgazzar AH, et al. Cyclic Cystography: Diagnostic yield in selected pediatric populations. Radiology 1999; 213(1):118-20.

11. Mahyar A, Ayazi P, MavadatiS, etal.Are clinical, laboratory, and imaging markers suitable predictors of vesicoureteral reflux in children with their first febrile urinary tract infection? Korean J Urol 2014;55(8):536-41.

12. Shah KJ, Robins DG, White RH. Renal scarring and vesicoureteric reflux. Arch Dis Child 1978;53(3):210-7.

13. Sciagrà R, Materassi M, Rossi V, etal.Alternativeapproaches to the prognostic stratification of mild to moderate primary vesicoureteral reflux in children. J Urol 1996; 155(6):2052-6.

14. Askari A, Belman AB. Vesicoureteral reflux in black girls. J Urol 1982; 127(4):747-8.

15. West W, Venugopal S. The low frequency of reflux in Jamaican children. Pediatr Radiol 1993; 23(8):591-3.

16. Caione P, Ciofetta G, Collura G, et al. Renal damage in vesico-ureteric reflux. BJU Int 2004; 93(4):591-5.

17. LeeJH, Son CH, LeeMS, etal. Vesicoureteral reflux increases 
the risk of renal scars: a study of unilateral reflux. Pediatr Nephrol 2006; 21(9):1281-4.

18. Peru H, Bakkaloğlu SA, Söylemezoğlu $\mathrm{O}$, et al. The relationship between urinary tract infections and vesicoureteral reflux in Turkish children. Int Urol Nephrol 2009; 41(4):947-51.

19. Bush NC, Keays M, Adams C, et al. Renal damage detected by DMSA, despite normal renal ultrasound, in children with febrile UTI. J Pediatr Urol 2015; 11(3):126.e1-7.

20. Roebock DJ, Howard RG, Metreweli C. How sensitive is ultrasound in the detection of renal scars? Br J Radiol 1999; 72(856):345-8
21. Donoso G, Lobo G, Arnello F, et al. Cicatriz renal detectada mediante cintigrama renal DMSA en niños con primera pielonefritis aguda: estudio de factores de riesgo. Rev Med Chile 2006; 134(3):305-11.

22. Garin EH, Olovarria F, Araya C, etal.Diagnosticsignificance of clinical and laboratory findings to localize site of urinary infection. Pediatr Nephrol 2007; 22(7):1002-6.

23. Anderson L, Preda I, Hahn-Zoric M, et al. Urinary proteins in children with urinary tract infection. Pediatr Nephrol 2009; 24(8):1533-8.

24. Pecile P, Miorin E, Romanello C, et al. Age-related renal parenchymal lesions in children with first febrile urinary tract infections. Pediatrics 2009; 124(1):23-9. 